\title{
Heteroduplex cleavage assay for screening of probable zygosities resulting from CRISPR mutations in diploid single cell lines
}

\author{
Kyle D. Luttgeharm, Kit-Sum Wong, and Steve Siembieda \\ Advanced Analytical Technologies, Inc., Ankeny, IA \\ BioTechniques 62:268-274 (June 2017) doi 10.2144/000114556 \\ Keywords: T7 endonuclease I; heteroduplex cleavage assay; CRISPR; gene editing; mutation detection \\ Supplementary material for this article is available at www.BioTechniques.com/article/114556.
}

The most common gene editing methods, such as CRISPR, involve random repair of an induced double-stranded DNA break through the non-homologous end joining (NHEJ) repair pathway, resulting in small insertions/deletions. In diploid cells, these mutations can take on one of three zygosities: monoallelic, diallelic heterozygous, or diallelic homozygous. While many advances have been made in CRISPR delivery systems and gene editing efficiency, little work has been done to streamline detection of gene editing events. The only current method to determine the zygosity of an edited gene in a diploid organism is DNA sequencing, which is costly and time-consuming. Here, we describe the development of a T7 endonuclease I (T7EI)-based heteroduplex cleavage assay, along with statistical models relating the percentage of cleaved DNA to the zygosity of a mutation, that provides a rapid screening step prior to DNA sequencing. By isolating candidates likely to contain the desired zygosity for the edited gene, our screening method can decrease the number of clones requiring DNA sequencing.

Clustered regularly interspaced short palindromic repeats (CRISPR)-based gene editing has led to an unprecedented increase in precise gene modifications. CRISPR gene editing uses the Cas9 endonuclease combined with a guide RNA (gRNA) to cleave a targeted genomic sequence and induce a double-stranded DNA break (1,2). After cleavage, cut DNA is repaired either by the non-homologous end joining (NHEJ) pathway or the homology directed repair (HDR) pathway. More commonly used, but error-prone, the NHEJ pathway randomly inserts or deletes DNA bases (indels) to repair the double-stranded break (3). The most common indels are \pm 1 or 2 bp, but larger indels can occur $(4,5)$. These modifications can result in a loss of gene function by introducing a frameshift mutation or a premature stop codon (6). Alternatively, during DNA repair the HDR pathway can insert a donor sequence with homology to sequences surrounding the cleavage site (7). While this method is effective for inserting specific nucleotide sequences, it is much less efficient overall than NHEJ and can involve complex procedures to limit NHEJ repair $(8,9)$. The increased use of CRISPR gene editing has led to many research groups working to improve the delivery and function of the CRISPR/Cas9 machinery (10); however, few have developed methodologies to rapidly screen for CRISPR mutations.

The simplest procedure for gene knockout is to perform CRISPR/Cas9 cleavage followed by the NHEJ repair pathway. The first step in the CRISPR/ Cas9 NHEJ mutation work flow involves delivery of Cas 9 and gRNA through either expression plasmids encoding both Cas9 and the gRNA, in vitro transcribed Cas9 mRNA and gRNA, or Cas9 protein complexed with gRNA (10). Regardless of which Cas9 delivery method is used, the cell pools must be screened for NHEJ events to determine mutation efficiency and to select cell pools for further study.
Following screening of initial pools, cells must be sorted and individual cell lines subsequently established. These individual cell lines are screened for the presence of a mutation, tested for the zygosity of the mutation, and then sequenced in order to determine the exact identity of the mutation (11). A single cell line with the desired mutation and zygosity is then identified for further research. A similar screening process is required for more complex multicellular organisms, such as mice, to identify and establish a mutant strain (9). CRISPR mutations in diploid cells can take on one of three possible zygosities. The simplest is a single mutation in one of the two alleles, referred to as a monoallelic mutation. When both alleles are mutated, this referred to as a diallelic mutation; when the mutations in both alleles are identical, this is referred to as a diallelic homozygous mutation, and when they are different, this is referred to as a diallelic heterozygous mutation.

\section{METHOD SUMMARY}

To increase the efficiency of identifying CRISPR gene editing mutations, we developed an optimized T7 endonuclease I-based heteroduplex cleavage assay that can predict the zygosity of an edited gene in diploid organisms. 
Statistical models have been developed to determine the percent of mutated alleles found in mutated pools of cells (12). However, methods to determine the zygosity of individual diploid cell lines and plants without DNA sequencing have yet to be developed. Given the increased use of CRISPR to edit diploid organisms, such as in induced human pluripotent stem cells and rodent models, a rapid, non-sequencing method for zygosity determination could save researchers time and money during the screening process.

Currently, sequencing remains the most reliable way to determine both the zygosity and the exact identity of the mutation. However, sequencing many lines can be both time-consuming and costly, resulting in the need for screening methodologies to identify the presence of gene editing events prior to sequencing. Different screening methodologies have been developed, including heteroduplex cleavage assays, high-resolution melting assays, qPCR, and heteroduplex mobility assays (13-15). While these methods can detect the presence or absence of a mutation, they have traditionally been limited in their abilities to determine all possible zygosity states of individual diploid cell lines. They also require subjective data interpretation, such as reading highresolution melting curves (16).

A heteroduplex cleavage assay can be used as a screening method, utilizing an endonuclease such as T7 endonuclease I (T7El) or Surveyor to identify and cleave mismatched DNA bases, resulting in a predictable fragmentation pattern (12,1719). The relative amount of fragments can be quantified and used to calculate percent cleavage and infer percent mutation in pooled cells, mosaic organisms, and individual cell lines/non-mosaic organisms (12). Previous research has focused on the differences between T7El's and Surveyor's abilities to detect gene editing events (18) as well as on the modification of endonuclease activity (20). T7El can detect a wide range of indels and all possible single nucleotide mismatches, while Surveyor prefers single nucleotide mismatches over small indels $(18,20)$. T7El has been shown to be sensitive enough to detect mutation rates $\geq 5 \%$, while the Surveyor endonuclease is unable to detect mutation rates $<10 \%$ (18).

Heteroduplex cleavage assays have been used to estimate the mutation rate in cell pools by relating the percent cleaved to the percent mutated (12). The percent mutated is calculated from the percent cleaved

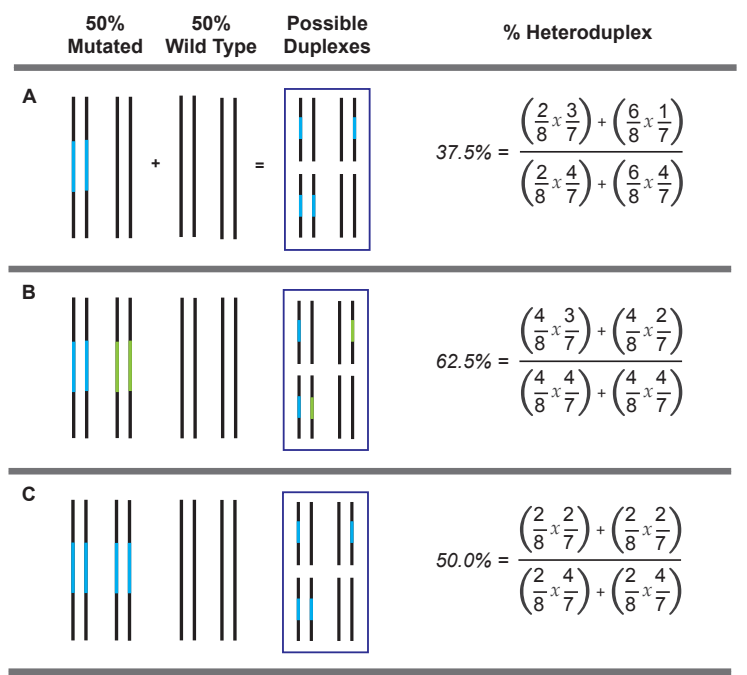

Figure 1. Graphical representation of the number of mutated alleles and the resulting duplexes when DNA from a CRISPR-edited diploid cell is mixed 50:50 (w/w) with wild type (WT) DNA and then heteroduplexed. The associated probability models for heteroduplex percentage are shown in the right column. (A) Monoallelic model where $25 \%$ of the DNA strands in the final mixture are mutated. (B) Diallelic heterozygous model where the DNA strands of the final mixture consist of $25 \%$ mutation $A$, $25 \%$ mutation $\mathrm{B}$, and $50 \%$ WT. (C) Diallelic homozygous model where $50 \%$ of the DNA strands in the final mixture are mutated.

using Equation 1, with the concentration of fragments expressed in molarity. While many publications use the terms percent cleaved and percent mutated interchangeably, it is important to note that the percent mutated is almost always lower than the observed percent cleaved. The percent mutated is calculated from the percent cleaved using the equations

\section{Percent Mutated $=1-(1-\text { Percent Cleaved })^{0.5}$}

and

$$
\text { Percent Cleaved }=\frac{\frac{C_{F 1}+C_{F 2}}{2}}{\frac{C_{F 1}+C_{F 2}}{2}+C_{A}}
$$

where $C_{F 1}=$ concentration of Fragment 1 , $C_{F 2}=$ concentration of Fragment 2 , and $C_{A}$ $=$ concentration of initial amplicon. Percent mutated represents the percent of mutated alleles in the total allele pool, not individual cells (12). Given the ability of heteroduplex cleavage assays to predict the number of mutated alleles in pooled cell lines, it is possible to use statistical models to predict the zygosity of individual cloned diploid cell lines and non-mosaic diploid organisms.

Despite the abundant research regarding endonucleases that target heteroduplexes, there has been no work on the ability of either T7El or Surveyor to produce percent cleavages consistent with the expected theoretical values. Here, we chose to investigate T7El due to its greater sensitivity and broad substrate preferences, particularly in relation to indel detection. Based upon the statistical probability of heteroduplex formation, we propose that the percent cleavage from heteroduplex cleavage assays can be used to infer the zygosity of a mutation prior to sequencing.

\section{Materials and methods}

Generation of mutant

plasmids and primers

A random 410 bp sequence named CRISPR model 1, with a GC content of $40 \%$, was generated and deemed the wild type (WT) version. The WT sequence was then modified to create sequences that contained all possible SNPs, along with $\pm 1, \pm 2$, and \pm 10 bp indels, and cloned into the EcoRV site of the pUC57 plasmid. Primers were designed using the pUC57 plasmid and synthesized sequences to amplify 300-1000 bp fragments with a GC content across the entire 1000 bp sequence of $50.4 \%$. Two additional 1000 bp sequences, CRISPR model 2 and CRISPR model 3, were synthesized to allow for amplicons of 3 unique sequences and GC contents across the 1000 amplified base pairs (CRISPR model 2: $42.2 \%$ GC; CRISPR Model 3: $32.5 \%$ GC). CRISPR models 2 and 3 were each modified to create -2 bp and +2 bp mutations. Primers were designed to amplify 300-1000 bp fragments of CRISPR models 2 and 3 using only the synthesized sequences. All sequences where synthesized by GenScript (Piscataway, NJ). Primer sequences can be found in Supplementary Tables 2-4.

\section{Genomic DNA extraction}

Genomic DNA was extracted from mature Kitaake Japonica Oryza sativa leaves using ZR Plant/Seed DNA MiniPrep (\#D6020; Zymo Research, Irvine, CA) per manufacturer's directions. 

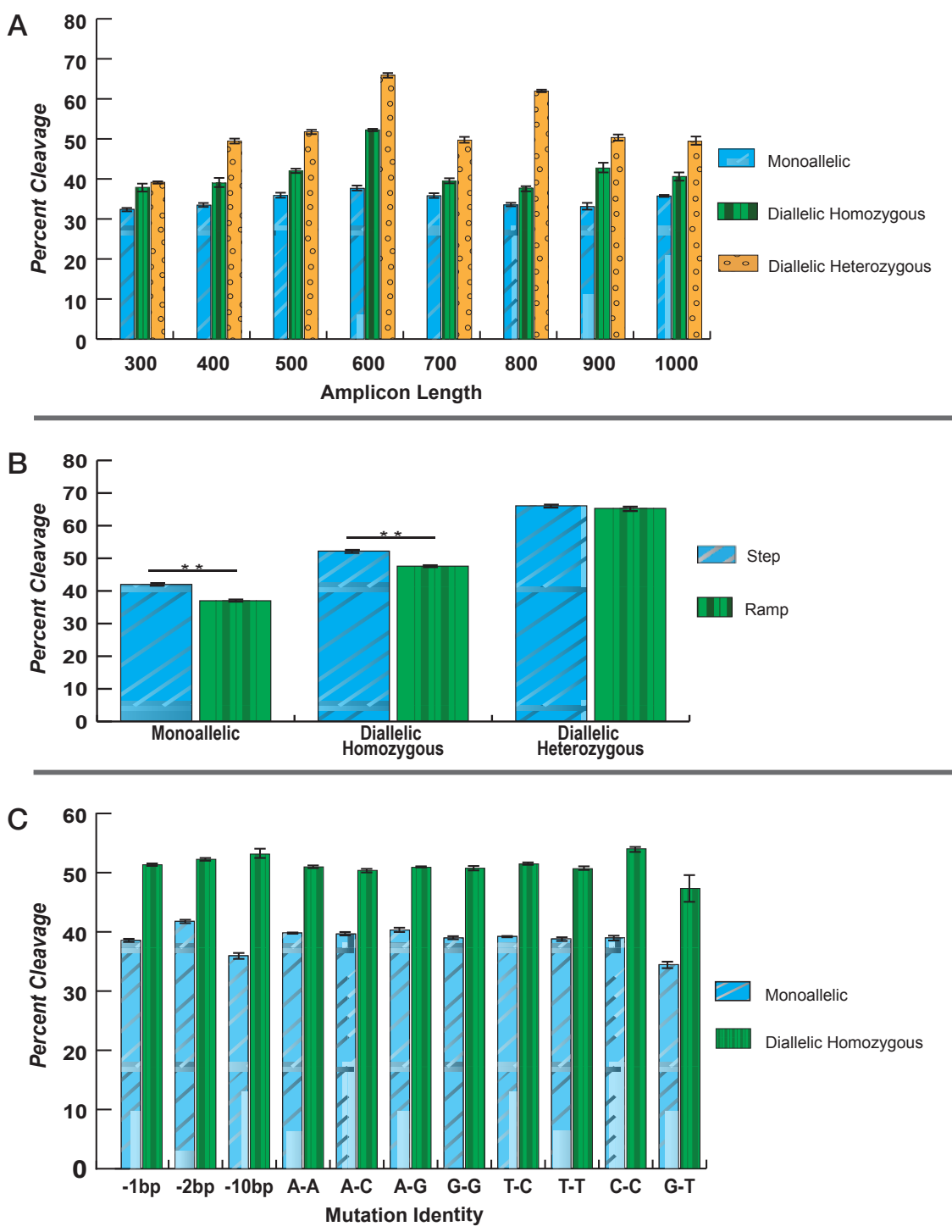

PCR amplification

All amplifications were performed using DreamTaq (\#EP0702, ThermoFisher Scientific, Waltham, MA). Briefly, all plasmid controls were amplified in either $25 \mu$ or 50 $\mu \mathrm{l}$ volumes with template concentrations of $0.5 \mathrm{ng} / 25 \mu \mathrm{l}$. O. sativa genomic DNA was amplified in a $50 \mu$ reaction volume using $20 \mathrm{ng}$ of template. All PCR reactions used
30 cycles $\left[95^{\circ} \mathrm{C}, 5 \mathrm{~min} ;\left(95^{\circ} \mathrm{C}, 30 \mathrm{~s} ; 54^{\circ} \mathrm{C}\right.\right.$, $30 \mathrm{~s} ; 72^{\circ} \mathrm{C} 1 \mathrm{~min}$ ) repeated; $72^{\circ} \mathrm{C}, 10 \mathrm{~min}$; $4^{\circ} \mathrm{C}$ hold] except for the $O$. sativa genomic extracts, which used 35 cycles using primers found in Supplemental Table S5.

\section{Heteroduplex formation}

After amplification, PCR products were quantified using the Qubit dsDNA HS Assay

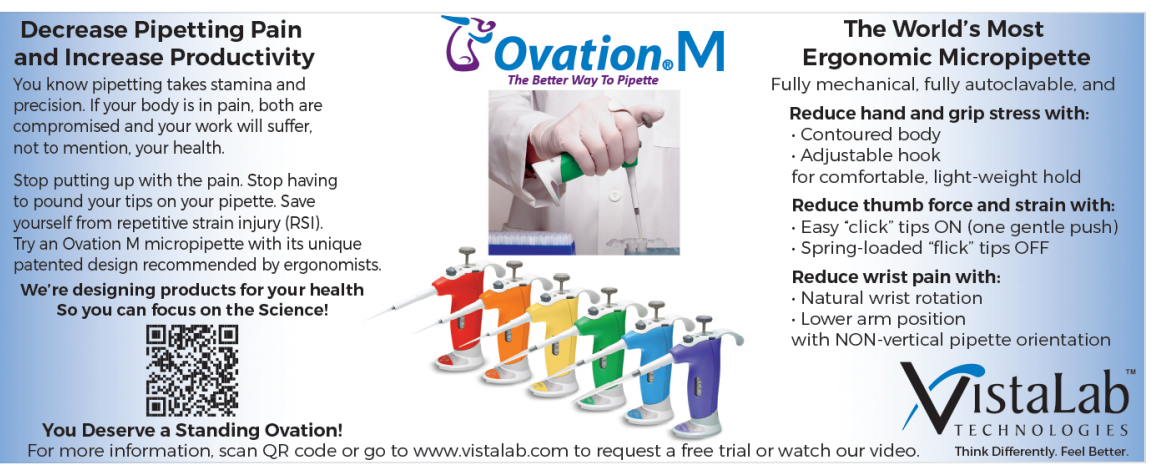

Figure 2. Optimization and validation of the T7 endonuclease I (T7EI) heteroduplex cleavage assay for zygosity prediction. (A) Comparison of cleavage efficiency for different amplicon lengths. The expected theoretical values for the monoallelic, diallelic homozygous, and diallelic heterozygous models are 37.5\%,50.0\%, and $62.5 \%$, respectively. All models used CRISPR model 1 plasmids. The monoallelic model is composed of $75 \%$ wild type (WT)/25\% -2 bp amplicons; the diallelic homozygous model is composed of $50 \%$ WT/50\% -2 bp amplicons; and the diallelic heterozygous model is composed of $50 \% \mathrm{WT} / 25 \%-2 \mathrm{bp} / 25 \%+2 \mathrm{bp}$ amplicons. Only the $600 \mathrm{bp}$ amplicon provided results that were within $5 \%$ of the expected value and were statistically different between the models $(P<0.01)$. Data are shown as mean \pm SEM $(n=$ 4). (B) Comparison of the Step and Ramp methods of heteroduplex formation. All models used CRISPR model 1 plasmids. The monoallelic model is composed of $75 \% \mathrm{WT} / 25 \%-2 \mathrm{bp}$ amplicons; the diallelic homozygous model is composed of $50 \%$ WT/50\% -2 bp amplicons; and the diallelic heterozygous model is composed of $50 \% \mathrm{WT} / 25 \%-2 \mathrm{bp} / 25 \%+2 \mathrm{bp}$ amplicons. No statistical difference was observed between the two heteroduplex formation methods in the diallelic heterozygous model, while a significant difference was observed between the heteroduplex formation methods for the monoallelic $(P<0.01)$ and diallelic homozygous model $(P$ $<0.01$ ). All zygosities were statistically different from each other within a particular heteroduplex formation method $(P<0.01)$. Data are shown as mean $\pm \operatorname{SEM}(n=4)$ and $* *$ as $P<0.01$. (C) Comparison of cleavage efficiency with different deletions and SNPs. All models used CRISPR model 1 plasmids. Monoallelic (75\% WT/25\% mutant) and diallelic homozygous (50\% WT/50\% mutated) models were analyzed for -1 bp, 2 bp, and 10 bp deletions as well as all possible SNPs. All mutations were statistically different between zygosity models at $P<0.01$ except for the G-T mismatch, which was significant at $P$ $<0.05$. Data are shown as mean $\pm \operatorname{SEM}(n=4)$.

Kit (\#Q32854; ThermoFisher Scientific). Plasmid mutation model amplicons were mixed at the desired ratio to model diallelic homozygous (WT/mutant, 1:1), diallelic heterozygous (WT/mutant,/mutant, $2: 1: 1$ ), or monoallelic mutations (WT/mutant, 3:1) in a total volume of $20 \mu$ with a final concentration of $10 \mathrm{ng} / \mathrm{\mu l}$ in $1 \times$ Cleavage Buffer (\#CP-CVB0001; Advanced Analytical Technologies, Inc, Ankeny, IA). 100 ng WT DNA and 100 ng CRISPR-edited DNA were mixed in a $20 \mu \mathrm{l}$ volume containing $1 \times$ Cleavage Buffer so that the final heteroduplex contained WT/edited DNA at a 1:1 ratio. Heteroduplex formation was completed by either the ramp method $\left(95^{\circ} \mathrm{C}, 10 \mathrm{~min} ; 95^{\circ} \mathrm{C}-85^{\circ} \mathrm{C}\right.$, decrease $2^{\circ} \mathrm{C} / \mathrm{s}$; $85^{\circ} \mathrm{C}-25^{\circ} \mathrm{C}$, decrease $0.1^{\circ} \mathrm{C} / \mathrm{s} ; 4^{\circ} \mathrm{C}$ hold) or the step method $\left(95^{\circ} \mathrm{C}, 10 \mathrm{~min} ; 94^{\circ} \mathrm{C}, 20 \mathrm{~s}\right.$; $93^{\circ} \mathrm{C}, 20 \mathrm{~s} ; 93^{\circ} \mathrm{C}-81^{\circ} \mathrm{C}$, decrease $2^{\circ} \mathrm{C}$ every $20 \mathrm{~s}$ for 6 cycles; $80^{\circ} \mathrm{C}, 20 \mathrm{~s} ; 79.7^{\circ} \mathrm{C}, 20 \mathrm{~s}$; $79.7^{\circ} \mathrm{C}-25^{\circ} \mathrm{C}$, decrease $0.6^{\circ} \mathrm{C}$ every $20 \mathrm{~s}$ for 92 cycles, ending at $\sim 25^{\circ} \mathrm{C} ; 4^{\circ} \mathrm{C}$ hold). 
T7 endonuclease I digestion

T7El digestion was performed using the AccuCleave T7CE Kit (\#DNF-440-1000CP; Advanced Analytical Technologies, Inc.) according to the manufacturer's instructions. Briefly, $4 \mu$ l heteroduplex DNA (40 ng) was mixed with $1 \mu \mathrm{l}$ T7El master mix (where $1 \mu \mathrm{l}$ contains $0.005 \mu \mathrm{l}$ T7El, $0.1 \mu \mathrm{l}$ Cleavage Buffer, and $0.9 \mu \mathrm{l}$ nuclease-free water) and then incubated at $37^{\circ} \mathrm{C}$ for $30-60 \mathrm{~min}$. The entire reaction was diluted with $200 \mu \mathrm{l} 0.1 \times$ TE and analyzed using Advanced Analytical Technologies' Fragment Analyzer CRISPR Mutation Discovery Gel Kit (\#DNF-910K1000CP). The percent cleavage was calculated using the CRISPR plugin for Advanced Analytical Technologies PROSize Data Analysis Software.

\section{Results and discussion}

To complement the design and delivery of gene editing techniques, advances in mutation detection techniques are needed for CRISPR/Cas9 mutation detection. The only current methodology to determine the zygosity of CRISPR-edited diploid organisms is DNA sequencing, which can be costly and time-consuming with deconvolution of sequencing reads often required. To overcome these limitations, we developed an endonuclease assay along with statistical models to relate the observed percent cleavage to the zygosity of a CRISPR-edited diploid cell.

\section{Development of statistical}

heteroduplex percent cleavage models To determine if it is possible to distinguish between monoallelic, diallelic heterozygous, and diallelic homozygous genotypes, we developed probability models for heteroduplex formation based upon the total number of strands and number of mutated DNA strands, as shown in Figure 1 (see also Supplementary Equation S1). Cells containing a diallelic homozygous mutation will produce non-cleavage homoduplexes after heteroduplexing that are indistinguishable from WT. Thus, addition of WT DNA at a ratio of $50 \%$ mutant $/ 50 \%$ WT is necessary to detect these mutations.

When monoallelic DNA is mixed 50/50 with WT DNA, the percentage of total mutated alleles in the sample will be 25\% (Figure 1A). The theoretical percent of heteroduplexes was determined by dividing the probability of heteroduplex formation with the probability of hetero- or homoduplex formation as shown in Figure $1 \mathrm{~A}$. The theoretical percentage of heteroduplex formation was found to be $37.5 \%$. Upon mixing of diallelic heterozygous and WT DNA at a 50/50 ratio, the percentage of the alleles will be: $25 \%$ mutation A, $25 \%$ mutation B, and 50\% WT (Figure 1B). The percent heteroduplex formation was found to be $62.5 \%$ using the same methodology as describe above. A simpler case was the mixing of $50 \%$ diallelic homozygous and $50 \%$ WT (Figure 1C), which gave 50\% heteroduplex formation.

\section{Comparison of amplicon length on cleavage efficiency}

To understand if amplicon length contributes to enzyme cleavage efficiency, amplicons ranging 300-1000 bp, increasing in 100-bp increments, were amplified for the diallelic homozygous, diallelic heterozygous, and monoallelic models, heteroduplexed following the ramp procedure, and then cleaved with T7El (Figure 2A). Only the 600-bp amplicon returned percent cleavages that were within $\pm 5 \%$ of the theoretical value. To further confirm these results, two additional gene models were tested, showing similar results (Supplementary Figure S1). Based on these data, all future experiments were conducted using a 600-bp amplicon.

\section{Comparison of heteroduplex}

formation procedures

To determine if the heteroduplex procedure impacts the percent cleavage, two different heteroduplexing protocols were compared (Figure 2B). Fragments 600-bp-long were amplified from 3 different plasmids, a WT plasmid, a 2-bp deletion, and a 2-bp insertion of the WT. Heteroduplexes were formed for the diallelic homozygous model by mixing amplifications of the $-2 \mathrm{bp}$ and WT plasmids 50:50 (w/w), for the diallelic heterozygous model by mixing amplicons of the $-2 \mathrm{bp},+2 \mathrm{bp}$, and WT plasmids 25:25:50 ( $\mathrm{w} / \mathrm{w})$, and for the monoallelic model by mixing amplicons of the $-2 \mathrm{bp}$ and WT plasmids 25:75 (w/w). All mixes were heteroduplexed using the ramping function of a thermocycler (Ramp Method) or by repeated small decreases in temperature followed by a 20 s hold (Step Method). The Ramp Method can be completed in 20 min, while the Step Method takes approximately $1 \mathrm{~h}$. Even though the two methods produced statistically different percent cleavage for the diallelic homozygous and monoallelic models, they were within $\pm 5 \%$

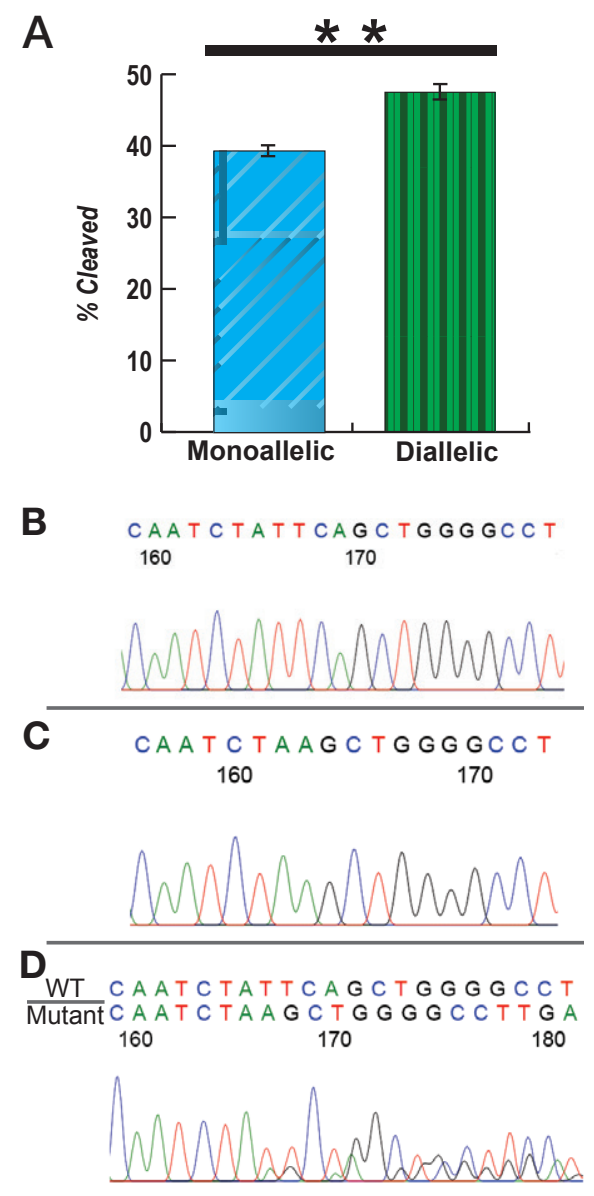

Figure 3. T7 endonuclease I (T7EI) heteroduplex cleavage from genomic DNA identifies monoallelic and diallelic homozygous events. Wild type (WT) O. sativa amplicons were heteroduplexed 50\%/50\% with CRISPR-edited amplicons and cleaved with T7EI. Plants containing probable monoallelic and diallelic homozygous events were identified $(A)$ and confirmed by Sanger sequencing: (B) WT; (C) diallelic homozygous; (D) monoallelic. The $-3 \mathrm{bp}$ deletion is underlined in red. Data are shown as mean \pm SEM, where monoallelic $n=12$, diallelic homozygous $n=11$, and ${ }^{* *}$ indicates $P<0.01$.

from their respective theoretical values. Despite the statistical difference of percent cleavage for the diallelic homozygous and monoallelic models using the 2 heteroduplexing methods, all zygosity models were statistically different among themselves $(P<0.01)$. Since both methods produced results within $\pm 5 \%$ of the theoretical percent cleavage of each model, all future heteroduplexing reactions were completed using the ramp method.

Cleavage efficiency of small indels and single nucleotide polymorphisms For the proposed methodology to be effective in determining the zygosity of diploid individuals, the heteroduplex cleavage assay must be able to cleave 
a variety of common CRISPR-induced mutations with similar efficiencies. To test this, amplicons representing $-1,-2$, and -10 bp indels, along with the SNPs A-A, A-C, A-G, C-C, G-G, G-T, T-C, and T-T, were heteroduplexed with WT amplicons using both the diallelic homozygous and monoallelic models previously described (Figure 2C). To determine the accuracy of the assays, the difference between the actual and theoretical values was calculated. All mutations for both the diallelic homozygous and monoallelic models were found to be within the $\pm 5 \%$ error window previously established and were significantly different between the 2 zygosity states at $P<0.01$, except for the G-T mismatch, which was statistically significant at $P<0.0295$.

\section{Amplification from genomic DNA}

To further verify the assay's usefulness, we confirmed the ability of T7EI to achieve theoretical percent cleavages from a segregating population of CRISPR-edited Kitaake Japonica Oryza sativa. Genomic DNA was amplified, followed by mixing of the PCR products 1:1 (WT:CRISPR-edited) to ensure heteroduplex formation. Upon T7El cleavage, probable monoallelic and diallelic homozygous lines were identified and subsequently confirmed by Sanger sequencing. Both the probable monoallelic and probable diallelic homozygous percent cleavages were found to be within the $\pm 5 \%$ error window (Figure $3 A$ ). Sanger sequencing determined that the probable monoallelic line contained a genotype of $-3 \mathrm{bp} / \mathrm{WT}$, and the probable diallelic homozygous line contained a genotype of $-3 \mathrm{bp} /-3 \mathrm{bp}$ (Figure 3, B-D).

Here, we present a heteroduplex cleavage assay that cuts a variety of mutations at expected values to determine zygosities. T7El was chosen due to its widespread use in detecting CRISPR mutations and previous reports of its ability to cleave both indels and singlebase mismatches $(17,18,20)$. The use of plasmid models allowed for the determination of T7El's exact substrate specificity. We found that T7El could cleave all single base mismatches as well as $\pm 1,2$, and 10 indels within $5 \%$ of the expected theoretical values. Interestingly, we did observe an amplicon size effect, with only the $600 \mathrm{bp}$ amplicon providing cleavage values consistent with those expected. Slight differences were observed when using different heteroduplex formation procedures for the monoallelic and diallelic homozygous models. However, regardless of the heteroduplex procedure used, the monoallelic and diallelic homozygous values were within $5 \%$ of the theoretical value. Additionally, every model was statistically different, thus not impacting the final result.

With the increased use of CRISPR/ Cas 9 gene editing, new methodologies are needed to rapidly screen a large number of events. Using T7El, we developed an assay that can be used to simultaneously determine both the presence of an induced mutation and its zygosity. The values for the different models and genomic DNA tested all fell within $5 \%$ of the expected value (monoallelic 38.5\% $\pm 5 \%$, diallelic homozygous $50 \% \pm 5 \%$, diallelic heterozygous $62.5 \% \pm 5 \%$ ), resulting in no overlap between the 3 different zygosity states. From the data, we proposed a $\pm 5 \%$ window from the theoretical percent cleavage for the determination of probable zygosities. While this technique cannot be used to definitively determine the zygosity of a sample, it can greatly reduce the number of sequencing reactions required, resulting in both time and cost savings.

\section{Acknowledgments}

The authors would like to acknowledge Bing Yang for the gift of CRISPR-edited Oryza sativa and Si Nian Char for creation of the edited Oryza sativa as well as Chava Pocernich and Benjamin Deist for editing this manuscript.

\section{Author contributions}

K.L. and S.S. conceived the study, and the experiments were designed by K.L. K.S.W. developed the heteroduplex probability models. All authors contributed to the data analysis. The manuscript was written by K.L. All authors reviewed the results and approved the final manuscript.

\title{
AccuCleave wotos \\ Detecting CRISPR gene editing events just got QUICKR
}

\author{
Introducing AccuCleave for use with \\ agarose gels
}

- Simple and accurate enzymatic digestion assay

- Reduce sequencing costs and times with efficient screening methods

- Optimized Taq eliminates time consuming PCR cleanup

Streamline your genetic editing workflows

at www.aati-us.com/AccuCleave

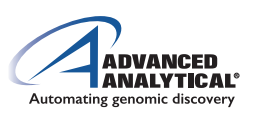




\section{Competing interests}

All authors are employees of Advanced Analytical Technologies, Inc. S.S. and K.S.W. have stock options in Advanced Analytical Technologies, Inc.

\section{References}

1. Cong, L., A. Ran, D. Cox, L. Shuailiang, R. Barretto, N. Habib, P.D. Hsu, X. Wu, et al. 2013. Multiplex genome engineering using CRIPSR/Cas systems. Science. 339:819-824.

2. Jinek, M., K. Chylinski, I. Fonfara, M. Hauer, J.A. Doudna, and E. Charpentier. 2012. A programmable dual-RNA-guided DNA endonuclease in adaptive bacterial immunity. Science 337:816-821.

3. Ran, F.A., P.D. Hsu, J. Wright, V. Agarwala, D.A. Scott, and F. Zhang. 2013. Genome engineering using the CRISPR-Cas9 system. Nat. Protoc. 8:2281-2308.

4. Pan, C., L. Ye, L. Qin, X. Liu, Y. He, J. Wang, L. Chen, G. Lu, et al. 2016. CRISPR/Cas9-mediated efficient and heritable targeted mutagenesis in tomato plants in the first and later generations. Sci. Rep. 6:24765.

5. Santiago, Y., E. Chan, P.-Q. Liu, S. Orlando, L. Zhang, F.D. Urnov, M.C. Holmes, D. Guschin, et al. 2008. Targeted gene knockout in mammalian cells by using engineered zinc-finger nucleases. Proc. Natl. Acad. Sci. USA 105:5809-5814.

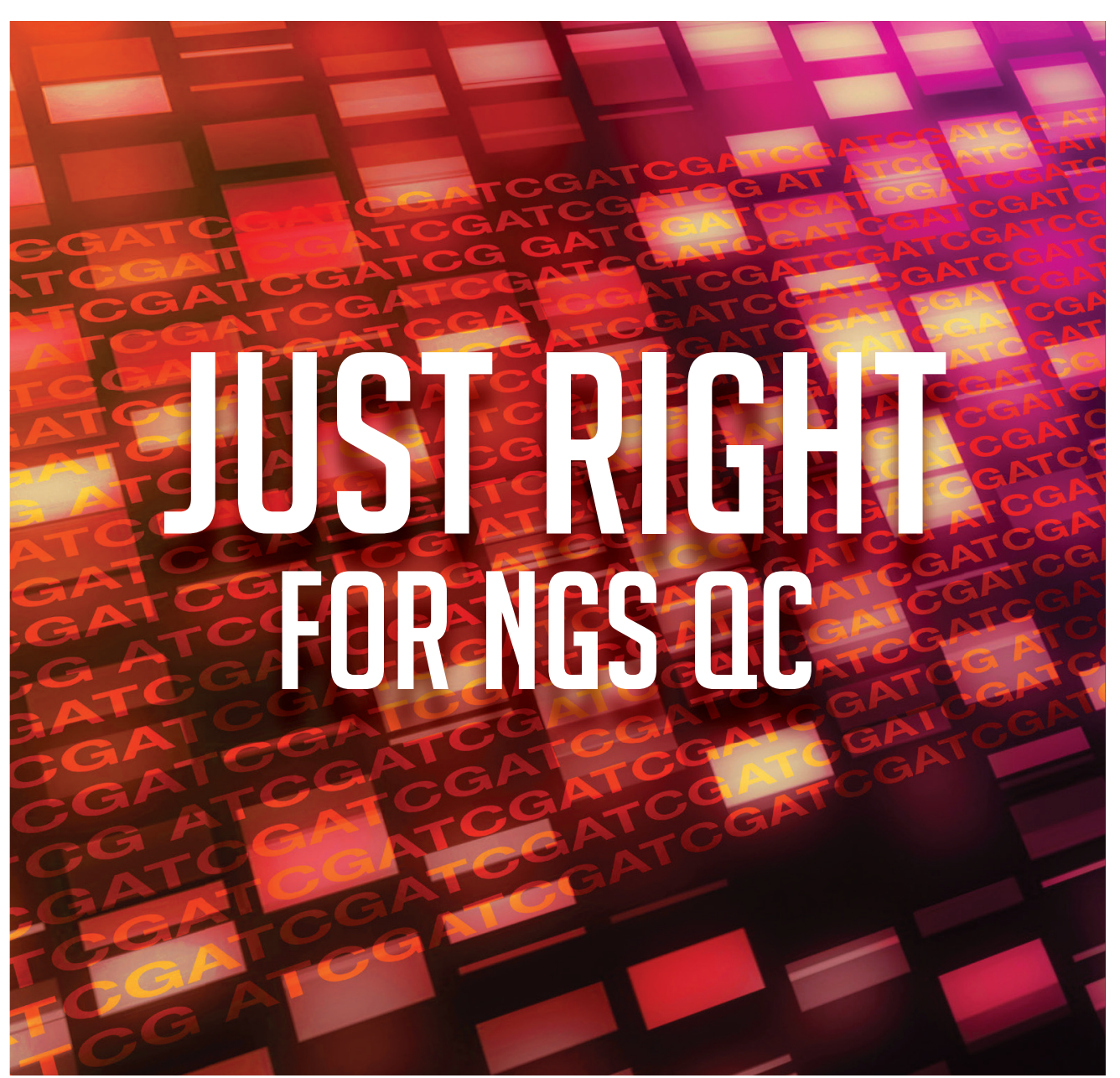

\section{AUTOMATE THE ANALYSIS OF NGS LIBRARY SMEARS UP TO 15,000 bp.}

Fragment Analyzer ${ }^{\mathrm{TM}}$ accurately sizes and quantifies typical NGS library smears - both large and small-by leveraging specially designed reagent kits.

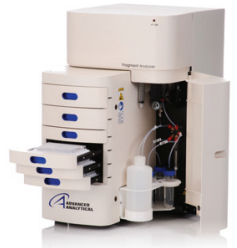

6. Perez, E.E., J. Wang, J.C. Miller, Y. Jouvenot, K.A. Kim, O. Liu, N. Wang, G. Lee, et al. 2008. Establishment of HIV-1 resistance in CD4+ T cells by genome editing using zinc-finger nucleases. Nat. Biotechnol. 26:808-816.

7. Gaj, T., C.A. Gersbach, and C.F. Barbas III. 2013. ZFN, TALEN, and CRISPR/Cas-based methods for genome engineering. Trends Biotechnol. 31:397-405.

8. Lin, S., B.T. Staahl, R.K. Alla, J.A. Doudna, R. Adams, J. Lindsay, J. Arron, M. Winslow, et al. 2014. Enhanced homology-directed human genome engineering by controlled timing of CRISPR/Cas9 delivery. eLife 3:e04766.

9. Wang, H., H. Yang, C.S. Shivalila, M.M. Dawlaty, A.W. Cheng, F. Zhang, and R. Jaenisch. 2013. One-step generation of mice carrying mutations in multiple genes by CRISPR/Cas-mediated genome engineering. Cell 153:910-918.

10. Sander, J.D. and J.K. Joung. 2014. CRISPRCas systems for editing, regulating and targeting genomes. Nat. Biotechnol. 32:347355.

11. Yang, L., J.L. Yang, S. Byrne, J. Pan, G.M. Church, L. Yang, J.L. Yang, S. Byrne, et al. 2014. CRISPR/Cas9-directed genome editing of cultured cells. Curr Protoc Mol Biol. 107:31.1.1-31.1.17.

12. Guschin, D.Y., A.J. Waite, G.E. Katibah, J.C. Miller, M.C. Holmes, and E.J. Rebar. 2010. A rapid and general assay for monitoring endogenous gene modification. Methods $\mathrm{Mo}$ Biol. 649:247-256.

13. Samarut, É, A. Lissouba, and P. Drapeau. 2016. A simplified method for identifying early CRISPR-induced indels in zebrafish embryos using High Resolution Melting analysis. BMC Genomics 17:547.

14. Zhu, X., Y. Xu, S. Yu, L. Lu, M. Ding, J. Cheng, G. Song, X. Gao, et al. 2014. An efficient genotyping method for genomemodified animals and human cells generated with CRISPR/Cas9 system. Sci. Rep. 4:6420.

15. Yu, C. 2014. A PCR based protocol for detecting indel mutations induced by TALENs and CRISPR/Cas9 in zebrafish. PLoS One 9:e98282.

16. Zischewski, J., R. Fischer, and L. Bortesi. 2017. Detection of on-target and off-target mutations generated by CRISPR/Cas9 and other sequence-specific nucleases. Biotechnol. Adv. 35:95-104

17. Babon, J.J., M. McKenzie, and R.G.H. Cotton. 2003. The use of resolvases T4 endonuclease $\mathrm{VII}$ and $\mathrm{T7}$ endonuclease I in mutation detection. Mol. Biotechnol. 23:73-81.

18. Vouillot, L., A. Thélie, and N. Pollet. 2015. Comparison of T7E1 and surveyor mismatch cleavage assays to detect mutations triggered by engineered nucleases. G3 (Bethesda) 5:407-415.

19. Mashal, R.D., J. Koontz, and J. Sklar. 1995. Detection of mutations by cleavage of DNA heteroduplexes with bacteriophage resolvases. Nat. Genet. 9:177-183.

20. Guan, C., S. Kumar, R. Kucera, and A. Ewel. 2004. Changing the enzymatic activity of $\mathrm{T} 7$ endonuclease by mutations at the $\beta$-bridge site: alteration of substrate specificity profile and metal ion requirements by mutation distant from the catalytic domain. Biochemistry 43:4313-4322.

Received 12 December 2016; accepted 20 April 2017

Address correspondence to Kyle D. Luttgeharm, Advanced Analytical Technologies, Inc., 2450 SE Oak Tree Ct. Suite 101, Ankeny, IA 50021. E-mail: kluttgeharm@aati-us.com 